\title{
Visual search for targets defined by combinations of color, shape, and size: An examination of the task constraints on feature and conjunction searches
}

\author{
PHILIP T. QUINLAN and GLYN W. HUMPHREYS \\ Birkbeck College, University of London, London, England
}

\begin{abstract}
A series of experiments are reported in which the comparative constraints on single-feature and conjunction searches were examined. The first three tested the idea that the critical differences between these searches reflect the number of stimulus attributes that subjects must extract to make a response, that is, one in the feature condition, two in the conjunction condition. Targets were defined by possible pairwise combinations of a color, a size, and a shape. In another condition, subjects searched for two simultaneously present feature targets. Search for these targets did not differ qualitatively from that for a single-feature target (i.e., search remained parallel), but there was a constant increment to feature search functions. The final experiment examined the possibility that the number of relevant shared features between targets and distractors may also be critical. The results showed that the number of relevant shared features affected the rate of search but not its nature. Thus, differences between feature and conjunction searches do not reflect the number of relevant stimulus attributes that must be detected, or the number of relevant features shared between targets and distractors. Nevertheless, evidence for attentional involvement was found when subjects searched for two simultaneously present features. This goes against the claim that differences between the two types of search occur because feature searches can be carried out preattentively, while only conjunction searches involve the serial application of focused attention. An account of visual search performance that emphasizes the fidelity of coding of target information seems a more adequate proposal.
\end{abstract}

The present experiments follow a tradition of visual search studies that have examined Treisman's ideas about selective attention and stimulus integration (Allport, Tipper, \& Chmiel, 1985; Bundesen \& Pedersen, 1983; Duncan, 1985; Egeth, Virzi, \& Garbart, 1984; Treisman, 1982; Treisman \& Schmidt, 1982; Treisman \& Souther, 1985; Treisman, Sykes, \& Gelade, 1977).

A detailed account of the ideas has been provided by Treisman and Schmidt (1982) in the form of the featureintegration theory of attention. The theory comprises a fairly comprehensive description of the role of attention in visual object recognition. Central to the theory is the idea that visual perception is characterized by two functionally independent and sequential stages. At the first stage, processing is preattentive: all separable features (Garner, 1974) are coded independently and in parallel, using populations of feature detectors for such dimensions as color, size, and shape, to form different feature maps (see, for instance, Attneave, 1950, for evidence demon-

\footnotetext{
This research was supported by grants from the Economic and Social Research Council. Experiment 4 arose out of suggestions made by John Duncan and Marge Eldridge. We would like to thank them and Nick Chmiel and Jane Riddoch for discussing their ideas and providing helpful comments on the research. Reprint requests should be sent to Philip Quinlan, Department of Psychology, Birkbeck College, University of London, Malet Street, London, WC1E 7HX, England.
}

strating the independent processing of size and form, and Handel \& Imai, 1972, for evidence demonstrating independent processing of color and form). Within each map, the presence of features is specified and their locations are coded in relation to one another. Furthermore, some preattentive segmentation and grouping is carried out whereby features within each map are combined into clusters. At the second stage of processing, crossdimensional integration takes place. For example, a particular color cluster may be combined with a particular shape cluster to give rise to the percept of a particular colored shape. The act of combining constituent features to form a conjunction of features is known as feature integration.

In Treisman's theory (Treisman \& Schmidt, 1982), feature integration can be determined by any one of three factors. First, feature integration may come about by the application of focused attention to each map location in a serial fashion. To provide a familiar, although mixed, metaphor, it is the "spotlight" of attention which provides the "glue" to stick features together.

Second, a feature conjunction may be perceived without the need for focused attention simply because it fits with expectations specified by particular object frames (Minsky, 1975). For instance, a subject may not need to attend to the location of a London taxi to perceive that 
it is black rather than another color. Finally, features may be randomly conjoined. It follows that with random conjoining, some features may be incorrectly integrated and, hence, that illusory conjunctions will be formed (see Treisman \& Schmidt, 1982). Although it is possible to generate hypotheses about the latter two means of feature integration, the first is of primary interest here.

The theory has been driven by a series of experimental results obtained from, in particular, two visual search tasks (Treisman, 1982; Treisman \& Gelade, 1980; Treisman et al., 1977). In one task (feature search), subjects search displays of colored letters for a target color or a target letter. In the other task (conjunction search), subjects search similar displays for a colored letter target. On half the trials, a single target occurs among the distractor letters; on the remaining trials, only the distractors occur. Across the trials, the number of letters in the displays varies.

The standard results for the feature-search task are that, on feature-present trials, there is a nonlinear relationship between speed of search and size of display. In fact, the search function may be relatively flat (see Treisman \& Gelade, 1980). On feature-absent trials, search times increase linearly with increases in display size. Moreover, there is a large difference in the slope values between the feature-present and the feature-absent functions. In the conjuction-search task, the search function for both present and absent trials describes linear increases in search times with increases in display size. The absent slope is typically about twice that of the present slope (Treisman \& Gelade, 1980; Treisman et al., 1977).

From this pattern of results, the following set of conclusions have been drawn. First, the pickup of critical feature information occurs in parallel. This is in keeping with the idea of an initial stage of parallel, preattentive processing. To explain the linearly increasing search functions of absent trials, Treisman et al. (1977) argue that a serial comparison process determines the "absent" responses. The suggestion is that two sorts of feature processing go on simultaneously; one is fast and parallel, the other is slower and serial. "Present" responses are made on the completion of the parallel processing, but "absent" responses are typically made on the completion of the serial processing.

In contrast to the feature searches, both conjunctionpresent and conjunction-absent responses depend on serial search, consistent with the operation of serial attention. "Present"' reponses are made as soon as a target is detected, otherwise every item in the display must be searched before an "absent" response can be made. The contrast between the serial self-terminating and the serial exhaustive searches gives rise to the 2:1 slope difference between absent and present search functions.

The inferred qualitative difference between parallel and serial search processes underlying feature and conjunction searches supports the functional separation of the two stages posited by the feature-integration theory.
Although feature-integration theory gives a consistent account of the findings of the two search tasks, it is important to note aspects of the experimental design which pose problems for making the connection between the theory and the data. Two factors will be considered here, and both concern the differing demand characteristics of the two search tasks. The first point is that the detection of a feature target depends upon the pickup of one item of information, whereas the detection of a conjunction target depends on the pickup of two items of information. By definition, subjects must pick up a color and shape to detect a color-shape conjunction, whereas they need pick up only a color or a shape to detect either feature target. Therefore, the difference between feature and conjunction searches may simply be a reflection of what happens when responses are based on the pickup of one versus two items of information. By this account, it is not the act of conjoining which is the critical factor, but merely that the response demand characteristics are different in the two search tasks. To test this possibility in the present study, subjects had to base responses on the detection of two features which were separated in the visual field. For example, subjects responded "present" only if both a target color and a target shape occurred in a display, and "absent" if neither was present or if only one was. This search task is known here as the both condition. By comparing performance in this condition with performance in the standard search conditions, it should become clear whether the feature-conjunction difference is due to the requirements of feature integration or to those involved in basing responses on two items rather than one item of information.

The both condition is also of interest because it provides an example of a search task in which performance with multiple targets is assessed (see Duncan, 1985, for a review). Duncan (1980), in a number of experiments, has persuasively demonstrated that the presence of simultaneous targets in search displays can profoundly affect search performance. Typically, the accuracy of target report decreases when more than a single target is present in search displays, whereas accuracy is not strongly affected when distractors are presented simultaneously with a single target. The performance decrements with multiple targets are consistent with the idea that target report is dependent on a serial, capacity-limited (attentional) process, which can operate only on one target at a time. The rejection of distractors, however, can operate in parallel (i.e., preattentively). Since the distinction between targets and distractors can be based on quite high levels of representation (animal vs. nonanimal; see Ostry, Moray, \& Marks, 1976), the suggestion is that higher order information, inciuding conjunction information, is represented preattentively. According to this account, only one target may be identified at a time, so we may expect some increases in search times in the both condition relative to the single-feature condition. The predictions of feature-integration theory are less clear. However, since 
feature information is presumed to be processed in parallel, and since the theory does not impose any constraints on feature selection, performance in the both condition should be no different from performance in the slowest, single-feature condition. That is, subjects search for both features in parallel, but their detection of both targets may then simply depend on the time needed to detect the slower feature. Whatever the case, the pattern of results in the both condition should throw some light on the mechanisms of target selection in visual search.

The second issue investigated here concerns the effect of the number of shared relevant attributes between targets and distractors on visual search. In typical singlefeature and conjunction searches, the number of shared relevant attributes between targets and distractors differs. This may mean that the level of discriminability between targets and distractors is higher in features searches than in conjunction searches. This is most easily explained by example. In the first experiment reported by Treisman et al. (1977), subjects in one feature-search condition searched for a dark pink $O$ in displays comprising purple and brown Os: thus, although the target shared its shape with all of the distractors, this attribute was not relevant to the task, since the target could be distinguished by its color. In the conjunction condition, the target was again a pink $\mathrm{O}$, but distractors were pink Ns and green Os. In this case, both color and shape were relevant to the task and all of the distractors shared one relevant attribute with the target. Thus, feature targets shared no relevant attributes with any of the distractors, whereas conjunction targets shared one relevant attribute with every distractor. The number of shared relevant attributes between targets and distractors covaried with the feature/conjunction difference.

Treisman et al. (1977) dismiss the possibility that shared relevant attributes is a critical variable in their search functions because of the contrast between the parallel and serial searches on present trials in the feature and conjunction conditions. They argue that the discriminability account would predict that the increase in search times with display size would be steeper for the conjunction condition than for the single-feature condition, but it could not explain why the search function is linear for conjunction searches but nonlinear for feature searches. However, despite their argument, it remains possible that the processes involved in generating serial (linear) search functions are quantitatively rather than qualitatively different from those involved in generating parallel (nonlinear) search functions. In particular, flat search functions may be obtained when a target is easily distinguished from its background, whereas serially increasing search functions may be obtained when targets and distractors are difficult

Table 1

Summary of the Conditions, Targets, and Distractors Used in Each Experiment

\begin{tabular}{|c|c|c|c|}
\hline Experiment & Condition & Targets & Distractors \\
\hline \multirow[t]{3}{*}{$\begin{array}{l}\text { Experiment } 1 \\
\text { (all small } \\
\text { letters) }\end{array}$} & $\begin{array}{l}\text { Single features: } \\
\text { color or shape }\end{array}$ & $\begin{array}{l}\text { blue }(\mathrm{A} \text { or } \mathrm{H}) \text { or } \\
\mathrm{C} \text { (orange or } \\
\text { green) }\end{array}$ & $\begin{array}{l}\text { green As } \\
\text { orange Hs }\end{array}$ \\
\hline & $\begin{array}{l}\text { Conjunction: } \\
\text { color/shape }\end{array}$ & green $\mathbf{H}$ & $\begin{array}{l}\text { green As } \\
\text { orange Hs }\end{array}$ \\
\hline & $\begin{array}{l}\text { Both features: } \\
\text { color and shape }\end{array}$ & $\begin{array}{l}\text { blue }(\mathrm{A} \text { or } \mathrm{H}) \text { and } \\
\mathrm{C} \text { (orange or } \\
\text { green) }\end{array}$ & $\begin{array}{l}\text { green As } \\
\text { orange Hs }\end{array}$ \\
\hline \multirow[t]{3}{*}{$\begin{array}{l}\text { Experiment } 2 \\
\text { (all letter } \mathbf{H} \text { ) }\end{array}$} & $\begin{array}{c}\text { Single features: } \\
\text { color or size }\end{array}$ & $\begin{array}{l}\text { blue (small or } \\
\text { medium) or LARGE }\end{array}$ & $\begin{array}{l}\text { small orange } \\
\text { medium green }\end{array}$ \\
\hline & $\begin{array}{l}\text { Conjunction: } \\
\text { color/size }\end{array}$ & small-green & $\begin{array}{l}\text { small orange } \\
\text { medium green }\end{array}$ \\
\hline & $\begin{array}{l}\text { Both features: } \\
\text { color and size }\end{array}$ & $\begin{array}{l}\text { blue (small or } \\
\text { medium) and LARGE } \\
\text { (orange or green) }\end{array}$ & $\begin{array}{l}\text { small orange } \\
\text { medium green }\end{array}$ \\
\hline \multirow[t]{3}{*}{$\begin{array}{l}\text { Experiment } 3 \\
\text { (all green } \\
\text { letters) }\end{array}$} & $\begin{array}{l}\text { Single features: } \\
\text { size or shape }\end{array}$ & $\begin{array}{l}\text { LARGE (A or } \mathrm{H} \text { ) or } \\
\mathrm{C} \text { (small or } \\
\text { medium) }\end{array}$ & $\begin{array}{l}\text { small As } \\
\text { medium Hs }\end{array}$ \\
\hline & $\begin{array}{l}\text { Conjunction: } \\
\text { size/shape }\end{array}$ & small $\mathbf{H}$ & $\begin{array}{l}\text { small As } \\
\text { medium Hs }\end{array}$ \\
\hline & $\begin{array}{l}\text { Both features: } \\
\text { size and shape }\end{array}$ & $\begin{array}{l}\text { LARGE ( } A \text { or } H) \\
\text { and } C \text { (small or } \\
\text { medium) }\end{array}$ & $\begin{array}{l}\text { small As } \\
\text { medium Hs }\end{array}$ \\
\hline \multirow[t]{2}{*}{ Experiment 4} & $\begin{array}{l}\text { One-feature } \\
\text { (conjunction) }\end{array}$ & small green $H$ & $\begin{array}{l}\text { medium, green As } \\
\text { medium, orange Hs } \\
\text { small, orange As }\end{array}$ \\
\hline & $\begin{array}{l}\text { Two-feature } \\
\text { (conjunction) }\end{array}$ & small green $\mathrm{H}$ & $\begin{array}{l}\text { small, green As } \\
\text { small, orange Hs } \\
\text { medium, green Hs }\end{array}$ \\
\hline
\end{tabular}


to distinguish. In one extreme case, targets share no relevant attributes with distractors; in the other extreme case, targets share all but one relevant attribute with distractors (see Duncan, 1985). Stated this way, the feature- and conjunction-search tasks may simply occupy opposite ends of a hypothetical search continuum.

We sought to investigate the effects of searching for two items rather than one item of information in a featuresearch condition, and, in addition, to learn how search performance might be affected by manipulation of the number of attributes shared by targets and distractors. The layout of this paper is as follows: First, a series of experiments (Experiments 1-3) are reported that examined feature and conjunction search using the possible pairwise combinations of color, shape, and size. In each experiment, a condition in which subjects responded "present" only following the detection of two features (the both condition) was also included. In this situation, when the features were present they were in separate locations in the displays. These three experiments analyzed search performance for two independent features and for conjunctions over a range of different features. They also provided the necessary baselines for the final experiment (Experiment 4), in which the effect of the number of shared features on search performance was investigated. In Experiment 4 , conjunctions were defined on the basis of three features (a particular letter of a particular color of a particular size), and performance was examined according to whether the distractors shared one or two features with the targets. For easy examination, the variables manipulated in each of the experiments are given in Table 1.

\section{GENERAL METHOD}

Unless otherwise stated, the same methods were used in Experiments 1-3.

\section{Subjects}

In each experiment, 12 subjects were selected from the Birkbeck College subject pool. They took part in a single experimental session lasting approximately $45 \mathrm{~min}$. Their ages ranged from late teens to early $30 \mathrm{~s}$, and all reported having normal or corrected-to-normal vision.

\section{Apparatus}

The presentation of the stimuli and the timing and data collection were under the control of an Apple II Europlus microcomputer. Two response keys were interfaced with the Apple, and reaction times, contingent on buttonpresses, were recorded by the Apple. All of the experimental material was displayed on the screen of a Skantic Luxor 14" monitor (Model 18037819). The monitor was viewed from a distance of $51 \mathrm{~cm}$.

In each experiment, the subjects searched for a given target against various arrays of colored letters. Across the four experiments, the letters $A, H$, and $C$ were used. All of the letters comprised straightline segments so that the A was made up of two long vertical lines joined by top and middle crossbars. The $C$ was essentially a left square bracket. In the various conjunction conditions, only the letters $A$ and $\mathrm{H}$ were used and the target was always the same small green $\mathrm{H}$. Overall, the total search area was about $20^{\circ}$ high $\times 26^{\circ}$ wide. Letter size was manipulated across the experiments, and the absolute size of the letters is included with the details of each experiment. In all cases, the size of the letters and of the total search area were somewhat larger than those used by Treisman and Gelade (1980).

\section{Design}

Each experiment was run as a within-subjects design, with each subject taking part in the three separate conditions during a single experimental session.

\section{Procedure}

Subjects were given a general introduction to the experiment and given detailed instructions as they were run through a series of demonstration trials.

The sequence of events on each trial was as follows: Following a blank screen, a fixation cross, positioned at the center of the search area, was displayed for $500 \mathrm{msec}$. At the offset of the cross, response timing was initiated and a search display was presented. This display remained until the Apple picked up a keypress. Timing stopped when the keypress was detected. Feedback contingent on this keypress was given by the word "CORRECT" or "INCORRECT" displayed at the bottom left-hand side of the screen. The feedback message remained on for approximately $1.5 \mathrm{sec}$.

Following the several trials used for demonstration purposes, the remaining trials of a block of 24 were used for practice. The subjects were told to respond quickly but not to sacrifice time for errors.

\section{EXPERIMENT 1: TARGETS DEFINED BY COLOR AND SHAPE}

\section{Method}

In all search conditions, subjects searched displays in which the distractors were green As and orange Hs. However, the subjects searched for different targets in the different conditions.

In the feature condition, the subjects responded "present" if either the letter $\mathrm{C}$ or the color blue occurred in the display. On half the trials in which it was present, the $C$ was colored green and on the remaining half it was colored orange. Similarly, on half the trials in which the color blue was present, a blue $\mathbf{H}$ occurred, and on the remaining half, a blue A occurred. There were 144 trials, and a target was present on half of them. The number of distractors present in a display varied across the trials. Distractor set sizes of $1,5,15$, and 30 letters occurred equally often in both present and absent trials. When present, the target acted as an extra item, so, for the present displays, the display sizes were $2,6,16$, and 31 items. ${ }^{1}$ There were six basic displays for each of the four distractor set sizes, and each of the six displays consisted of a different random configuration of distractors. Across the six displays, the position of the target was varied randomly. The set of 48 displays (6 present, 6 absent for the four distractor set sizes) was presented three times to each subject. The total number of present trials for each set size was 18 , and each feature (i.e., $\mathrm{C}$ or blue) occurred nine times for each set size.

In the conjunction condition, the target was a green $\mathrm{H}$ and, as before, the distractors were green As and orange Hs. In the both condition, the subjects had to search for the presence of both the letter C and the color blue. The subjects had to respond "present" when both the target letter and the target color appeared in the same display, even though these two features always occurred in separate locations. The procedure in the both condition was the same as for single-feature and conjunction searches, except that present displays were constructed by adding the two targets to the configuration of distractors used on corresponding absent displays. The two target 
positions were randomly chosen and as far as possible the positions of the permissible pairs of targets were balanced across trials.

There were three types of absent trials in the both condition (24 trials each). The first set of absent trials, namely neither trials, were those on which neither target occurred. In the second (color-only) set of absent trials, the target color was present but not the target shape. In the third (shape-only) set of absent trials, the target shape was present but not the target color. The position of these single targets was allocated on a random basis.

Across the four general sorts of trials in the both condition (i.e., one present and three absent types of trials), an attempt was made to control for display size by altering the number of distractors. On present trials, the four distractor set sizes of $1,3,13$, and 28 served to provide overall display sizes of $3,5,15$, and 30 items. For neither trials, displays of $1,5,15$, and 30 distractors were used. Finally, for the remaining absent trials (i.e., in which only one target feature was present), there were distractor set sizes of $1,4,14$, and 29.

The order of the three search conditions (i.e., single feature, conjunction, and both features) was balanced across subjects, and each subject received a different random order of trials in each of the conditions. Half the subjects responded "present" with the preferred hand; the remaining responded "present" with the nonpreferred hand.

\section{Results}

Separate one-way repeated measures analyses of variance were carried out on both the response time (RT) and error data from each condition.

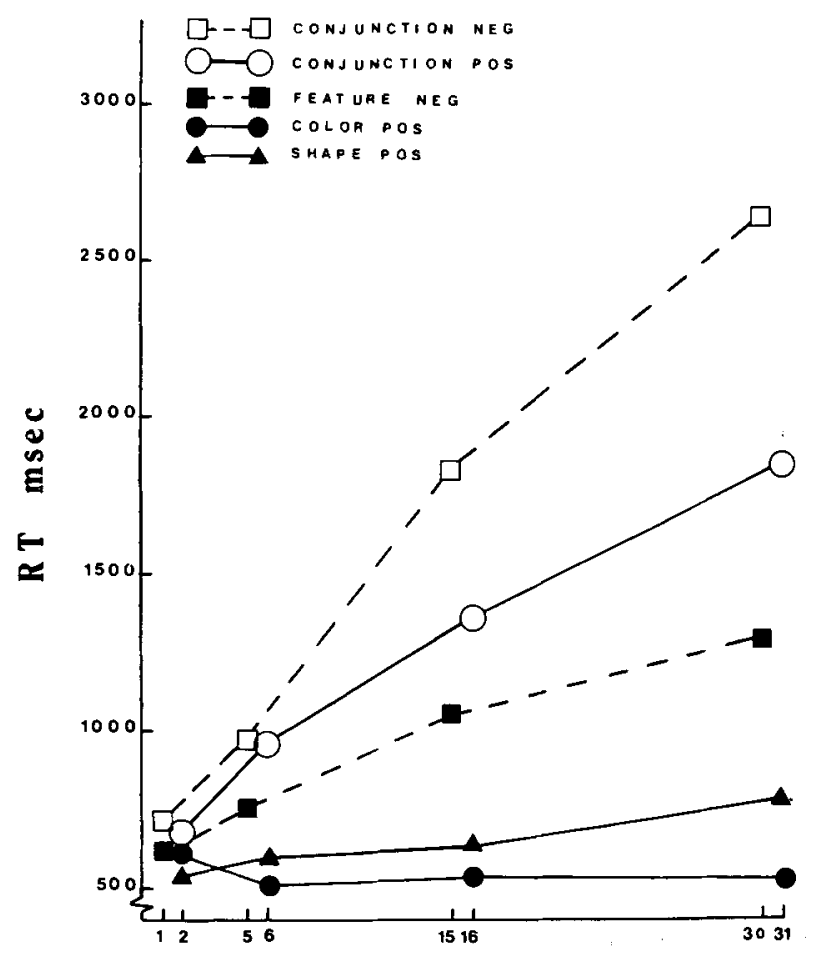

Display Size

Figure 1. Search times for feature and conjunction targets in Experiment 1. POS = target present; NEG = target absent.
Table 2

Linear Regressions of Reaction Time on Display Size for Experiment 1: Targets Defined by Color and Shape

\begin{tabular}{lccc}
\hline Condition & $\begin{array}{c}\text { Slope } \\
\text { (msec/item) }\end{array}$ & $\begin{array}{c}\text { Intercept } \\
\text { (msec) }\end{array}$ & $\begin{array}{c}\text { Percentage of } \\
\text { Variance } \\
\text { Accounted For by } \\
\text { Linear Component }\end{array}$ \\
\hline \multicolumn{4}{c}{ Feature Searches } \\
Color-present & -1.4 & 566 & $21.8^{*}$ \\
Shape-present & 7.4 & 540 & $98.4 \dagger$ \\
Feature-absent & 23.2 & 629 & $97.1 \dagger$ \\
& Conjunction Searches & \\
Conjunction-present & 38.9 & 675 & $98.1 \dagger$ \\
Conjunction-absent & 67.4 & 678 & $98.4 \dagger$ \\
\hline
\end{tabular}

${ }^{*}$ Linear component significant, $p<.05$. Hlinear component significant, $p<.01$.

Two questions were of primary interest. The first concerned whether the various search functions were strictly linear or whether there were reliable departures from linearity. The second concerned the ratio of slopes of the present and absent search functions. Consequently, only the results of the analyses relevant to these questions are reported. Speed-accuracy tradeoffs are discussed only when there was a main effect of display size on the error data. Also, given the close relationship between the first three experiments, discussion of the individual experiments is postponed until after the presentation of all three sets of results.

Feature search. Figure 1 shows the mean correct RTs for present and absent trials in both feature and conjunction searches.

For color-present responses, the search function departed from linearity $[F(1,11)=6.88, p<.05$, for the quadratic component; $F(1,11)=8.44, p<.01$, for the cubic component] and was relatively flat (see Figure 1).

The shape-present search function showed a significant linear trend $[F(3,33)=15.21, p<.001]$, which accounted for $98.4 \%$ of the variance. The search rate, given by the slope of this linear trend, was only $7.4 \mathrm{msec} / \mathrm{item}$ (see Table 2).

For absent responses, the linear component accounted for $97.1 \%$ of the variance, but both the quadratic $[F(1,11)$ $=20.97, p<.001]$ and the cubic $[F(1,11)=11.03$, $p<.01]^{2}$ components were also significant. The slope ratio for feature-absent relative to shape-present trials was 0.32: the slope for the feature-absent trials was over three times that for shape-present trials. ${ }^{3}$

There were no signs of any speed-accuracy tradeoffs in any of the above conditions. With increases in display size, error rates in the color-present condition were $5.5 \%$, $1.8 \%, 1.8 \%$, and $0 \%$, respectively; in the shape-present condition, they were $3.7 \%, 2.7 \%, 2.7 \%$, and $6.4 \%$; and in the feature-absent condition, they were $.9 \%, .4 \%, 0 \%$, and $0 \%$.

Conjunction search. For both conjunction-present and conjunction-absent responses there were strong effects of 
display size on RTs. The function for present responses was strictly linear, and this component accounted for $98.1 \%$ of the variance $[F(1,11)=66.18, p<.001]$. Least squares analysis showed that the search rate was approximately $40 \mathrm{msec} /$ item (see Table 2 ).

The search function for the conjunction-absent data departed from linearity $[F(1,11)=11.56, p<.001$, for the quadratic component; $F(1,11)=64.57, p<.001$, for the cubic component]. From the least squares analysis, the search rate in this condition was $67 \mathrm{msec} / \mathrm{item}$. The slope ratio of present to absent trials was 0.58 .

Error rates for all displays were far fewer in the conjunction-absent condition than in the conjunctionpresent condition. There were $3.7 \%, 3.2 \%, 7.8 \%$, and $6.4 \%$ errors for $1,5,15$, and 30 display sizes, respectively. Error rates for the conjunction-present condition were $4.2 \%, 5.1 \%, 10.6 \%$, and $23.6 \%$ for display sizes of $2,6,16$, and 31 items. There were again no signs of any speed-accuracy tradeoffs.

Both condition. Mean search times for the various types of trials are plotted as a function of display size in Figure 2.

There was a reliable effect of display size on the time taken to detect that both the target color and target shape were present. Only the linear component reached sig-

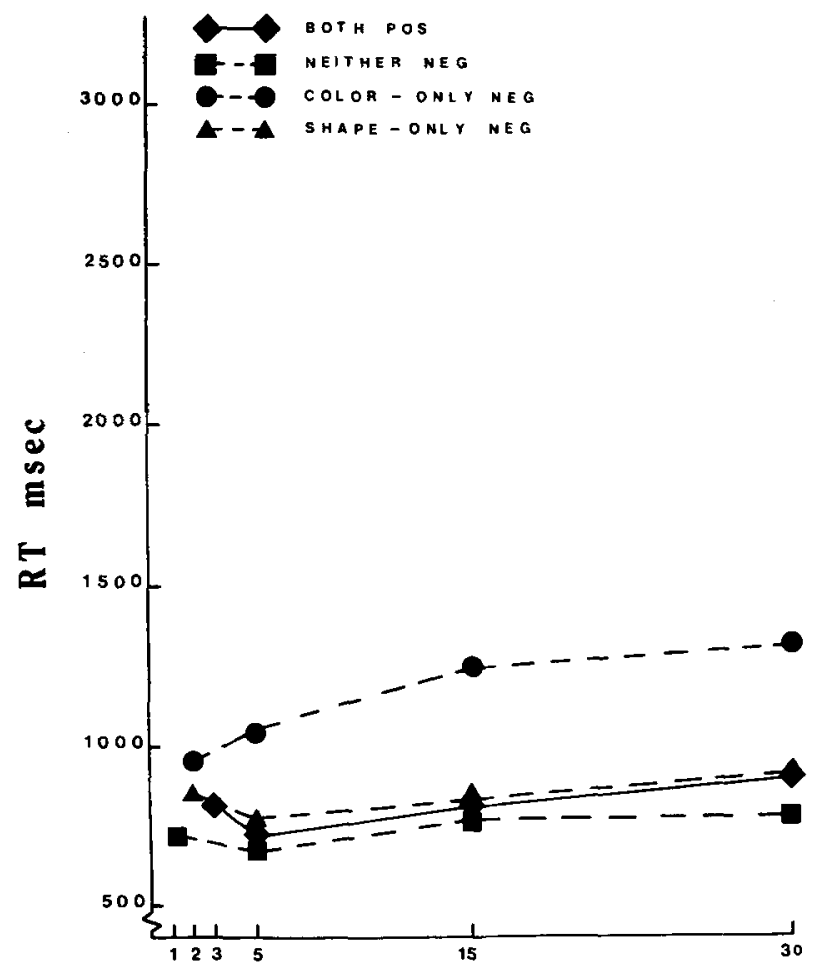

Display Size

Figure 2. Search time for targets in the both condition in Experiment 1. POS = targets present; NEG = target(s) absent.
Table 3

Linear Regressions of Reaction Time on Display Size for Both Searches in Exeperiment 1: Targets Defined by Color and Shape

\begin{tabular}{lccc} 
Condition & $\begin{array}{c}\text { Slope } \\
\text { (msec/item) }\end{array}$ & $\begin{array}{c}\text { Intercept } \\
\text { (msec) }\end{array}$ & $\begin{array}{c}\text { Percentage of } \\
\text { Variance } \\
\text { Accounted for by } \\
\text { Linear Component }\end{array}$ \\
\hline Both-present & 7.1 & 684 & $98.7^{*}$ \\
Both-absent & 0.2 & 633 & n.s. \\
Color-only & 28.5 & 827 & $98.9^{*}$ \\
Shape-only & 1.5 & 633 & n.s. \\
\hline
\end{tabular}

Note-n.s. $=$ linear component not significant, $p>.05$. *Linear component significant, $p<.01$.

nificance $[F(1,11)=18.36, p<.01]$, and it accounted for $98.7 \%$ of the variance. The search rate was approximately $7 \mathrm{msec} /$ item (see Table 3 ).

Error rates of $3.2 \%, 1.8 \%, 2.3 \%$, and $5.0 \%$ were recorded for display sizes of $3,5,15$, and 30 , respectively. There was no evidence of a speed-accuracy tradeoff.

Absent responses can be divided into three types: (1) neither target present ("'neither" responses), (2) color present but shape absent ("color-only" responses), and (3) shape present but color absent ("shape-only" responses). Data for each of these responses were analyzed separately.

On trials when neither target was present and on trials when only the shape target was present, RTs were unaffected by increases in display size (see Figure 2). In contrast, on color-only trials, RTs increased linearly with increases in display size $[F(1,11)=40.11, p<.001$, for the linear component]. The ratio of the slopes of the RT-display-size functions for the both-present to the color-only (absent) trials was 0.25 (see Table 3).

There were no signs of any speed-accuracy tradeoffs in any of the above conditions. With increases in display size, error rates in the "neither" response condition were $2.8 \%, 1.0 \%, 0 \%$, and $0 \%$, respectively; in the shape-only condition, they were $6.9 \%, 1.3 \%, 2.8 \%$, and $1.3 \%$; and in the color-only condition, they were $8.3 \%, 8.3 \%$, $12.5 \%$, and $11.1 \%$.

Summary. Search for single-feature targets was not serial and self-terminating. The function for a colordefined target was essentially flat, and, although a linear search function was obtained for shape-defined targets, the shape-present to feature-absent slope ratio was 0.32 . Functions closer to those expected if search were serial and self-terminating occurred for conjunction targets (see Discussion of Experiments 1-3).

Most importantly, the search function in the bothpresent condition resembled that found in the featuresearch condition on shape-present trials. When both feature targets were present (respond "present") and when only the color target was present (respond "absent"), the corresponding search functions were linear. The presentto-absent slope ratio was, however, only .25 . When neither target was present and when only the shape tar- 
get was present, responses were unaffected by increases in display size.

\section{EXPERIMENT 2: TARGETS DEFINED BY COLOR AND SIZE}

\section{Method}

Displays comprised only Hs and three sizes of letters were used. These were designated small, medium, and large. Small Hs were identical in size to the letters used in Experiment 1. Medium Hs were constructed with a $7 \times 14$ matrix of pixels and measured $0.6 \mathrm{~cm}$ wide $\times 1.2 \mathrm{~cm}$ high $\left(41^{\prime} \times 1^{\circ} 23^{\prime}\right)$. Finally, large $\mathrm{Hs}$ were constructed within a $11 \times 25$ matrix of pixels and measured $1.0 \mathrm{~cm}$ wide $\times 2.2 \mathrm{~cm}$ high $\left(1^{\circ} 9^{\prime} \times 2^{\circ} 31^{\prime}\right)$.

In all three search conditions (see below), small orange $\mathrm{Hs}$ and medium green Hs acted as distractors. Targets differed across the three conditions. In the feature condition, the subjects were to respond "present" if either a large $\mathbf{H}$ or a blue $\mathbf{H}$ occurred. Large Hs were colored green or orange; blue Hs were either small or medium. A shorthand way of describing this is to say that the target color was blue and the target size was large.

The single target in the conjunction condition was a small green $\mathrm{H}$. In the both condition, the subjects were to respond "present" if both a blue $H$ and a large $H$ occurred in the same display. As in the feature condition, the target color was blue and the target size was large.

\section{Results}

Feature search. Figure 3 shows the mean correct search times in the feature- and conjunction-search conditions plotted as a function of display size.

The search functions on color-present trials and on sizepresent trials both departed from linearity $[F(3,33)=$ $3.17, p<.05$, for the quadratic component in the colorpresent condition; $F(1,11)=7.50, p<.05$, for the cubic component in the size-present condition].

With absent responses, the search function was strictly linear $[F(1,11)=9.79, p<.01]$, and this component accounted for $76.8 \%$ of the variance. The slope and intercept values for the best-fitting straight lines for the feature and conjunction searches are shown in Table 4. The search rate in the feature-absent condition was approximately $3 \mathrm{msec} / \mathrm{item}$.

There were no signs of any speed-accuracy tradeoffs on the various types of trials in the feature-search condition. With increases in display size, error rates for the color-present trials were $3.7 \%, 2.7 \%, 1.0 \%$, and $0 \%$, respectively; for the size-present trials, they were $0.9 \%$, $6.4 \%, 3.7 \%$, and $3.7 \%$; and for the feature-absent trials, they were $1.3 \%, 1.3 \%, 1.3 \%$, and $2.3 \%$.

Conjunction search. For both conjunction-present and conjunction-absent trials, RTs increased linearly as a function of display size $[F(1,11)=45.16, p<.001 ; F(1,11)$ $=101.30, p<.001$, respectively, for the linear components]. The amount of variance accounted for by the linear components of the present and absent functions was $99.6 \%$ and $99.2 \%$, respectively. For present trials, the rate of search was $19.1 \mathrm{msec} / \mathrm{item}$; for absent trials, the rate was $34.9 \mathrm{msec} / \mathrm{item}$. The present-to-absent slope ratio was 0.55 .

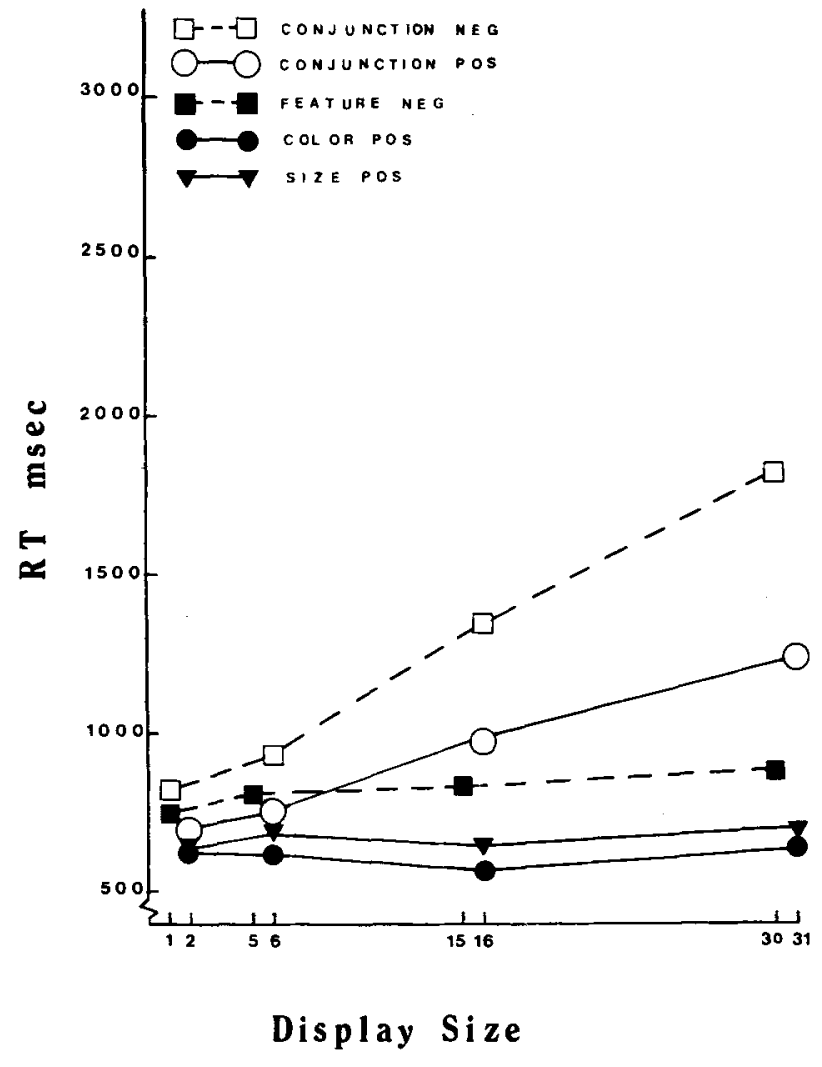

Figure 3. Search times for feature and conjunction targets in Experiment 2 . POS $=$ target present; NEG $=$ target absent.

On present trials, error rates were $1.4 \%, 0.5 \%, 8.3 \%$, and $14.8 \%$ for display sizes $2,6,16$, and 31 items, respectively. On the absent trials, there were $2.8 \%, 0.9 \%$, $1.4 \%$, and $2.3 \%$ errors to displays of $1,5,15$, and 30 items, respectively. There is no indication of any speedaccuracy tradeoffs.

Both condition. When both targets were present, RTs described a nonlinear relationship with display size $[F(1,11)=16.05, p<.001$, for the quadratic component; $F(1,11)=15.64, p<.001$, for the cubic component]. Figure 4 shows the mean search times for all types

Table 4

Linear Regressions of Reaction Time on Display Size for Experiment 2: Targets Defined by Color and Size

\begin{tabular}{|c|c|c|c|}
\hline Condition & $\begin{array}{c}\begin{array}{c}\text { Slope } \\
\text { (msec/item) }\end{array} \\
\end{array}$ & $\begin{array}{c}\text { Intercept } \\
\text { (msec) }\end{array}$ & $\begin{array}{c}\text { Percentage of } \\
\text { Variance } \\
\text { Accounted For by } \\
\text { Linear Component }\end{array}$ \\
\hline \multicolumn{4}{|c|}{ Feature Searches } \\
\hline $\begin{array}{l}\text { Color-present } \\
\text { Size-present } \\
\text { Feature-absent }\end{array}$ & $\begin{array}{l}0.1 \\
1.9 \\
3.4\end{array}$ & $\begin{array}{l}616 \\
643 \\
781\end{array}$ & $\begin{array}{l}\text { n.s. } \\
61.3^{*} \\
76.8^{*}\end{array}$ \\
\hline \multicolumn{4}{|c|}{ Conjunction Searches } \\
\hline $\begin{array}{l}\text { Conjunction-present } \\
\text { Conjunction-absent }\end{array}$ & $\begin{array}{l}19.1 \\
34.9\end{array}$ & $\begin{array}{l}656 \\
762 \\
\end{array}$ & $\begin{array}{l}99.6^{*} \\
99.2^{*}\end{array}$ \\
\hline
\end{tabular}

Note-n.s. $=$ linear component not significant, $p>.05 . \quad{ }^{*}$ Linear component significant, $p<.01$. 


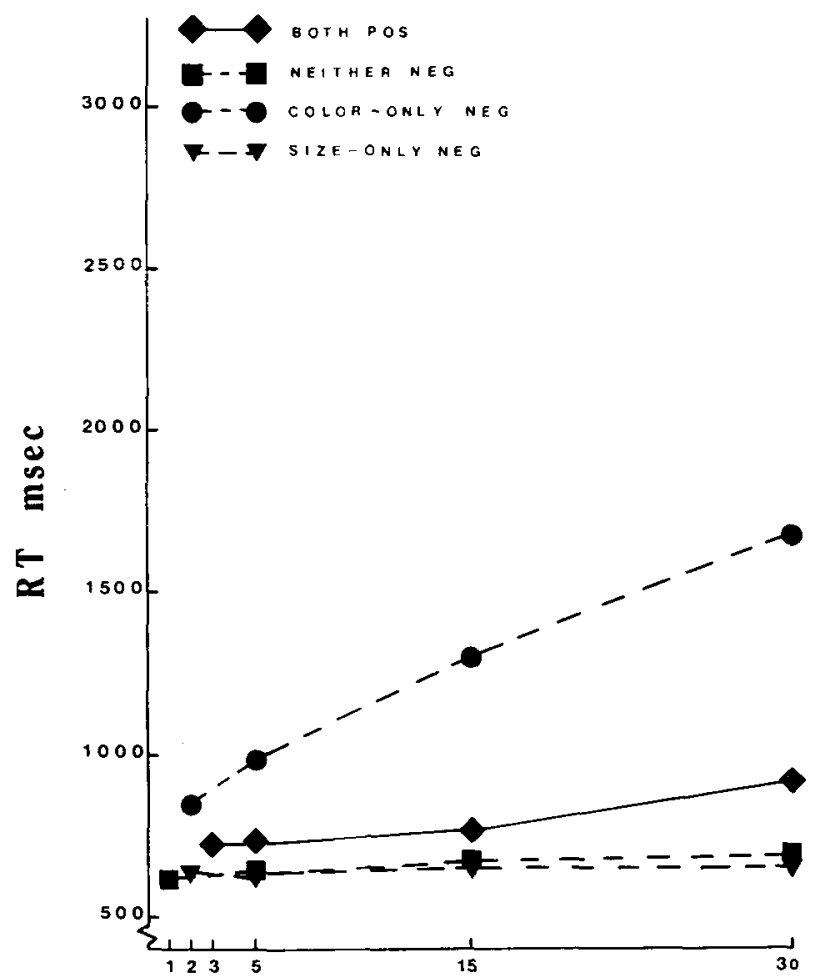

\section{Display Size}

Figure 4. Search times for targets in the both condition in Experiment 2. POS = targets present; NEG = target(s) absent.

of trials making up the both condition. Table 5 shows the slope and intercept values of the best-fitting straight lines calculated for the various conditions. The slope value for the both-present trials indicates that the estimated speed of search was approximately $4 \mathrm{msec} / \mathrm{item}$.

There were $3.2 \%$ errors for the responses to displays of 3 and 30 items and $2.3 \%$ and $1.3 \%$ errors to displays of 5 and 15 items. The errors showed a nonlinear pattern similar to that of the RTs.

Absent trials were again classed into three types, namely, neither, color only, and size only.

For neither and size-only RTs, there were no effects of display size on performance. However, for color-only responses, there was a reliable linear trend $[F(1,11)=$ $67.38, p<.001]$, which accounted for $84.1 \%$ of the variance. The approximate speed of search on these trials was $11 \mathrm{msec} / \mathrm{item}$ (see Table 5).

With increases in display size, error rates for neither responses were $3.2 \%, 2.3 \%, 1.3 \%$, and $3.2 \%$, respectively; for color-only trials they were $6.9 \%, 4.1 \%, 9.7 \%$, and $8.3 \%$, respectively; and for the shape-only trials, they were $5.6 \%, 1.4 \%, 1.4 \%$, and $0 \%$, respectively.

Summary. In the feature-search condition, flat search functions obtained for those trials on which a singlefeature target was present. On feature-absent trials, search times increased linearly with increases in display size.
Search in the conjunction condition was serial and selfterminating. Both conjunction-present and conjunctionabsent functions described linear relationships with increases in display size and the present-to-absent slope ratio was approximately 0.5 .

In the both condition, present responses were affected by increases in display size, but the search function departed from linearity. When both targets were absent (i.e., on neither trials), and when only the size target was present, the search function was essentially flat. However, on trials on which only the color target was present, RTs varied directly with display size.

\section{EXPERIMENT 3: TARGETS DEFINED BY SIZE AND SHAPE}

\section{Method}

Color was made irrelevant to the task by presentation of only green letters. The three sizes of letters used in Experiment 2 were again employed. In all three search conditions, the distractors were small As and medium-sized Hs. In the feature-search condition, the subjects searched for the presence of the target shape $C$ or the presence of the target size "large." Half the time C was present, it was small; on the remaining present trials, it was medium sized. On half the trials, when a large target was present it was an $\mathrm{A}$; on the remaining half, it was an $\mathbf{H}$.

In the both condition, the subjects were to detect the presence of a $\mathrm{C}$ and a large letter; in the conjunction condition, the single target letter was small green $\mathbf{H}$.

\section{Results}

Feature search. Figure 5 shows the mean search times in the feature- and conjunction-search conditions, plotted as a function of display size. Table 6 shows the regression statistics of the various search functions.

For the size-defined target, there was no effect of increasing display size on search times.

No errors were made to displays of 31 items. Errors of $4.6 \%, 0.9 \%$, and $6.4 \%$ were recorded for displays of 2,6 , and 16 items, respectively. There is no evidence of a speed-accuracy tradeoff.

For the shape-present condition there was a statistically significant main effect of display size on speed of search, and only the linear component reached significance $[F(1,11)=7.74, p<.05]$. The linear component accounted for $98.2 \%$ of the variance. The rate of search

Table 5

Linear Regressions of Reaction Time on Display Size for Both Searches in Experiment 2; Targets Defined by Color and Size

\begin{tabular}{lccc}
\hline Condition & $\begin{array}{c}\text { Slope } \\
\text { (msec/item) }\end{array}$ & $\begin{array}{c}\text { Intercept } \\
(\mathrm{msec})\end{array}$ & $\begin{array}{c}\text { Percentage of } \\
\text { Variance } \\
\text { Accounted For by } \\
\text { Linear Component }\end{array}$ \\
\hline Both-present & 3.9 & 766 & $52.6^{*}$ \\
Both-absent & 1.7 & 708 & n.s. \\
Color-only & 11.2 & 990 & $84.1^{*}$ \\
Size-only & 3.1 & 792 & n.s. \\
\hline
\end{tabular}

Note-n.s. linear component not significant, $p>.05$. *linear component significant, $p<.01$. 


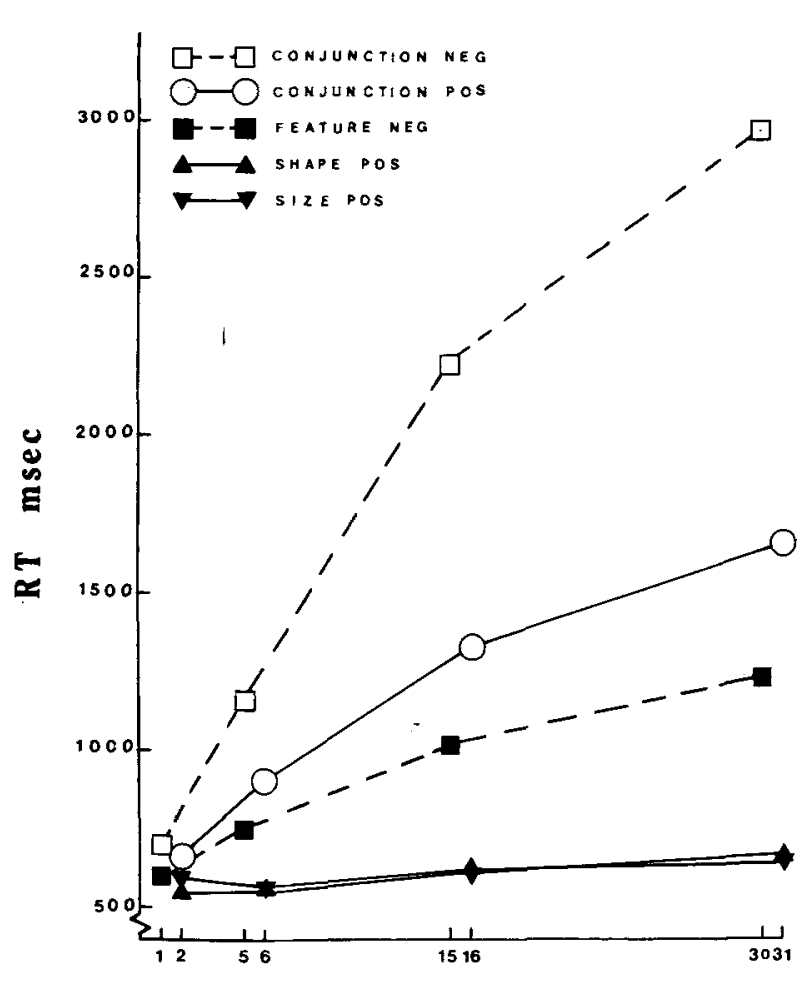

Display Size

Figure 5. Search times for feature and conjunction targets in Experiment 3. POS $=$ target present; NEG = target absent.

for the shape target in this experiment was only $3.5 \mathrm{msec} / \mathrm{item} .{ }^{4}$ Error rates were $2.3 \%, 1.9 \%, 0.4 \%$, and $0.4 \%$ for displays of $2,6,16$, and 31 items, respectively.

The search function for feature-absent responses departed from linearity $[F(1,11)=7.19, p<.05$, for the quadratic component]. This result is not due to a speedaccuracy tradeoff, however. Error rates were $2.3 \%$, $1.9 \%, 0.4 \%$, and $0.4 \%$ for display sizes $1,5,15$, and 30 items, respectively.

The ratio of the slope functions for shape-present to feature-absent responses was .16, a value that indicates an approximate sixfold difference in search rate in the two conditions.

Conjunction search. For present responses, the search function departed from linearity $[F(1,11)=14.12$, $p<.01$, for the quadratic component]. However, this seems likely to have been due to a speed-accuracy tradeoff. For the four display sizes used, corresponding error rates were $1.3 \%, 3.7 \%, 18.9 \%$, and $25.9 \%$. There were large increases in errors at the larger display sizes, which indicates that the subjects tended to respond "absent" if they failed to detect the conjunction target within a certain critical duration.

The search function for absent responses again departed from linearity $[F(1,11)=56.4, p<.001$, for the quadratic component; $F(1,11)=5.13, p<.05$, for the cubic component]. Nevertheless, the present-to-absent slope ratio was 0.44 , close to the theoretically critical ratio of 0.5 .

Error rates in the conjunction-absent condition were $5.1 \%$ for displays of $1 \mathrm{item}, 3.24 \%$ for displays of 5 items, $3.7 \%$ for displays of 15 items, and $0.93 \%$ for displays of 30 items. There was no evidence for a speedaccuracy tradeoff: accuracy increased linearly as the display size increased.

Both condition. Regression statistics for the various search functions are presented in Table 7. Figure 6 shows the various RT-display-size functions for all the searches.

With both-present responses, RTs showed a nonlinear relationship with display size $[F(1,11)=8.71, p<.05$, for the quadratic component]. Inspection of the error data, however, suggested a possible speed-accuracy tradeoff. The actual error rates were $2.3 \%, 3.2 \%, 0.9 \%$, and $6.0 \%$ for displays of $3,5,15$, and 30 items, respectively.

Absent responses were divided into the same general three categories as in Experiments 1 and 2 . In the neither and size-only conditions, there were reliable nonlinear effects of display size on performance $[F(1,11)=9.24$, $p<.05$, for the quadratic component in the neither search function; $F(1,11)=16.15, p<.05$, for the quadratic component in the size-only search function]. For shape-only responses, there was a significant linear effect of display size on speed of search $[F(1,11)=20.06$, $p<.001$ ], with $90.9 \%$ of the variance being accounted for.

For neither responses, errors were made only to displays comprising 1 and 30 items. The rates were $2.7 \%$ in both cases. For shape-only responses, the error rates for displays of $2,5,15$, and 30 items were $6.9 \%, 1.9 \%$, $2.7 \%$, and $1.4 \%$, respectively. For size-only responses, the corresponding error rates were $8.3 \%, 2.7 \%, 2.7 \%$, and $1.4 \%$. There were no speed-accuracy tradeoffs.

Summary. The results show that the search function for size in the feature condition was essentially flat and search times were relatively fast. Search for shape, however, produced a linearly increasing RT-display-size function. The search function for feature-absent trials departed significantly from linearity, with search times being relatively fast for the largest displays. The shape-

Table 6

Linear Regressions of Reaction Time on Display Size for Experiment 3: Targets Defined by Size and Shape

\begin{tabular}{|c|c|c|c|}
\hline Condition & $\begin{array}{c}\text { Slope } \\
\text { (msec/item) }\end{array}$ & $\begin{array}{c}\text { Intercept } \\
\text { (msec) }\end{array}$ & $\begin{array}{c}\text { Percentage of } \\
\text { Variance } \\
\text { Accounted For by } \\
\text { Linear Component }\end{array}$ \\
\hline \multicolumn{4}{|c|}{ Feature Searches } \\
\hline $\begin{array}{l}\text { Size-present } \\
\text { Shape-present } \\
\text { Feature-absent }\end{array}$ & $\begin{array}{r}1.9 \\
3.5 \\
21.9\end{array}$ & $\begin{array}{l}583 \\
551 \\
632\end{array}$ & $\begin{array}{l}\text { n.s. } \\
98.2^{*} \\
95.3 \dagger\end{array}$ \\
\hline \multicolumn{4}{|c|}{ Conjunction Searches } \\
\hline $\begin{array}{l}\text { Conjunction-present } \\
\text { Conjunction-absent }\end{array}$ & $\begin{array}{l}33.7 \\
77.2\end{array}$ & $\begin{array}{l}673 \\
771\end{array}$ & $\begin{array}{l}95.9 \dagger \\
96.9 \dagger\end{array}$ \\
\hline
\end{tabular}

Note-n.s. $=$ linear component not significant, $p>.05$. "Linear component significant, $p<.05$. †Linear component significant, $p<.01$. 
Table 7

Linear Regressions of Reaction Time on Display Size for Both Searches in Experiment 3: Targets Defined by Size and Shape

\begin{tabular}{lccc}
\hline Condition & $\begin{array}{c}\text { Slope } \\
\text { (msec/item) }\end{array}$ & $\begin{array}{c}\text { Intercept } \\
\text { (msec) }\end{array}$ & $\begin{array}{c}\text { Percentage of } \\
\text { Variance } \\
\text { Accounted For by } \\
\text { Linear Component }\end{array}$ \\
\hline Both-present & 5.9 & 648 & $88.6 \dagger$ \\
Both-absent & 6.9 & 634 & $65.1^{*}$ \\
Size-only & 23.4 & 728 & $93.4 \dagger$ \\
Shape-only & 9.0 & 767 & $90.9 \dagger$ \\
\hline
\end{tabular}

*Linear component significant, $p<.05$. †Linear component significant, $p<.01$.

present-to-feature-absent slope ratio was .16. Thus, there is no evidence that search for features is serial and selfterminating.

Departures from linearity were also obtained with present and absent responses in the conjunction condition. However, on conjunction-present trials, subjects appeared to be operating with self-imposed deadlines, as indicated by the speed-accuracy tradeoff. Nevertheless, inspection of the slope ratio of the best-fitting straight lines of these functions revealed a value of 0.44 , which is indicative of serial, self-terminating search.

In the both condition, every search function except the shape-only function departed from linearity. Search per-

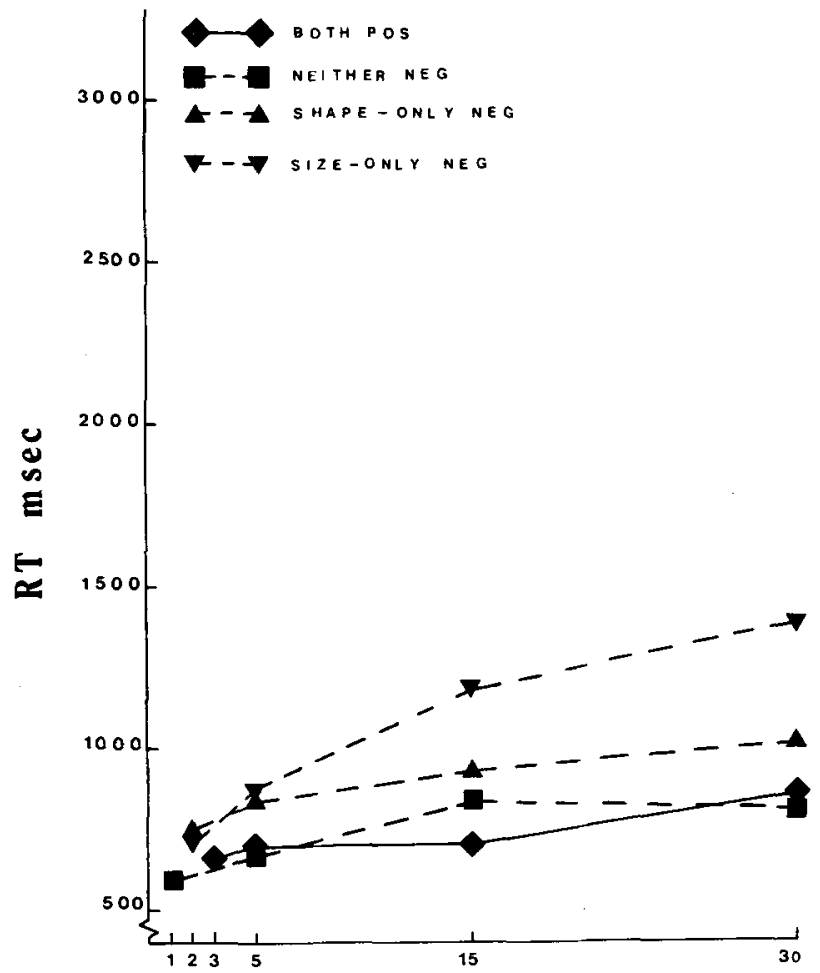

$$
\text { Display Size }
$$

Figure 6. Search times for targets in the both condition in Experiment 3. POS = targets present; NEG $=$ target(s) absent. formance did not conform to a serial, self-terminating pattern.

\section{DISCUSSION OF EXPERIMENTS 1, 2, AND 3}

The data from each feature search in Experiments 1-3 support the idea of a parallel search process. Search functions for present responses to targets defined by color and size were either flat or departed from linearity. Present RTs to shape-defined targets increased linearly as a function of the display size, but even so present-to-absent search ratios never approximated the theoretically critical value of 0.5 . This contrasts with the pattern of results found with conjunction-defined targets, where the presentto-absent slope ratios approximated 0.5 . The data suggest that conjunction targets were detected by means of a serial, self-terminating search.

One difference between the results reported here and those in previous studies is that responses on absent trials in both the feature and conjunction conditions sometimes departed from linearity. Search functions in these cases were negatively accelerated. This evidence is consistent with the idea that, on absent trials, subjects treated some of the larger displays as if they comprised smaller clusters of items. Locally defined clusters could be searched in parallel, with a serial processing component introduced by subjects' having to conduct a number of such searches across the display. Information about item clusters may play less of a part in searches of smaller display sizes because the items are then more widely spaced and are therefore less likely to form groups. In the latter case, a search of every item may be completed prior to the availability of information concerning item clusters. This may also mean that clustering effects will be more prevalent with absent than with present responses. Now, in order for clustering to be at all useful, information defining clusters ought to make explicit whether or not a target is present. When the target is present, its actual identity need not then be specified so long as it is grouped with an otherwise homogeneous cluster of items. Responses may then be based on group information which specifies either that a group is consistent (i.e., the target is absent) or that it is inconsistent (i.e., the target is present) (see Humphreys, Riddoch, \& Quinlan, 1985, for further evidence on this point).

\section{Both Searches}

The results from the both condition go against the hypothesis that the contrast between feature and conjunction search is due to the difference between basing responses on one versus two items of information. If this were correct, then search in the both condition ought to be equivalent to search in the conjunction condition. However, the results from all three experiments contradict this prediction. In only one experiment (in Experiment 1 where subjects had to pick up both a color and shape to respond "present") did the both-present search function 
have a reliable linear component. Furthermore, in all three experiments, when both targets were absent, all corresponding search functions were nonlinear and none of the slope ratios of the linear components of the functions even approximated 0.5 . Search in these conditions was clearly not serial and self-terminating.

With disconfirmation of the initial assumptions about performance in the both condition, it is useful to consider accounts that are more consistent with the results.

One trend, present in the results of all of the experiments, is that absent responses in the both condition were fast and relatively flat when displays did not contain the most salient feature (i.e., color in Experiments 1 and 2 and size in Experiment 3). This suggests that in the both condition subjects could terminate search on failing to find the most salient feature. However, when the most salient feature was present, responding was contingent on the pickup of the less salient feature.

Further inspection of the data suggests that although the search rates in the both-present and the slowest singlefeature-present searches were similar, overall RTs in the both-present condition were slower: that is, there appear to be intercept but not slope differences between the corresponding functions. Such a result is important when considered against the predictions of specific models of performance. It is useful to examine three such models. The first is a simple "horse-race" model in which subjects respond "present," in the both condition, on the completion of processing the slowest feature. Providing there is some overlap in the distribution of processing times for the two features, RTs in the both condition should be slower than those on the slowest feature trials in the feature search condition. This follows because processing of the slowest feature will sometimes be completed first. Now, given that increases in display size slows performance for both features, we may expect there to be a greater overlap in the distribution of the completion times for the slowest and both feature conditions at the larger display sizes. This follows if the effect of increasing the display size is to shift RTs at the slow rather than the fast end of the RT distributions. Consequently, the difference between the both and the slowest feature conditions should change multiplicatively as the display size increases-an effect which should show up in the slopes of the two associated search functions.

A second model holds that performance in the both condition is based on sequential ordering of the search for each feature. For instance, subjects might search first for the most salient feature target, and, having found that target, then begin to search for the least salient target. This predicts that the time to respond "both present" would be some combination of the search rates for the two features. When there are reliable effects of display size on performance, the slope of the both function should be steeper than the slope of the function for the slowest feature. This follows given that the slope of the both function will contain a component due to the initial search for the more salient feature.
A third model holds that search for the two types of feature target in the both condition takes place in parallel, but that the detection of the targets operates serially. In this case, the search rates in the both condition and in the slowest feature condition should be equivalent. However, there may be some constant increment in RTs due to another nonsearch process, such as the time to switch attention from the more salient feature to the less salient feature. This attention-switching process would show up as a difference in intercept values between the search functions for the both condition and the slowest feature condition.

To test the above predictions, it is important to assess performance when display size produced reliable effects on search in the feature conditions. This occurred in Experiments 1 and 2 but not in Experiment 3 (where there were no display-size effects on size-present responses). Also, direct comparisons of performance in the both and slowest feature conditions on present trials are problematic because the display size in the two conditions differed. Therefore, comparisons were based on the slope and $y$ intercepts for the both-present and slowest feature functions. The data from Experiment 1 are most appropriate, since the shape-present and the both-present conditions each described linear functions. For each subject, slope and intercept values were calculated for each of the above conditions. There was no difference beweeen the slope values for the shape-present and the both-present functions $(t<1.0)$. On the other hand, $y$-intercepts for bothpresent responses were greater than those for the shapepresent responses $[t(11)=3.69, p<.01$, two-tailed]. This finding of intercept but not slope effects is consistent with the idea that search for the features in the both condition proceeds in parallel. The one problem with this analysis is that the variance in the RTs in the color-present condition did not increase across the display sizes ( $S D$ s were $136,76,110$, and $101 \mathrm{msec}$ for display sizes 2,6 , 16 , and 31 , respectively). This contradicts the assumption that the overlap in the distribution of processing times for the two features will increase across the display sizes-a critical assumption upon which to test the horserace model. For this reason, we conducted a second analysis on absent responses in the single-feature search condition and in the size-only and the shape-only search conditions (i.e., "absent" responses in the both condition). Also, there was a considerable overlap in the distribution times for the size-only-absent and the feature-absent trials, and this overlap increased with display size [the means and the $S D$ s for the feature-absent trials were 758 (228), 1,029 (486), and 1,228 (636) msec, for display sizes $5,15,30$, respectively; for the size-only-absent trials, the corresponding statistics were $869(237), 1,178$ $(4,245)$, and $1,381(585) \mathrm{msec}]$. The contrast between the search functions for the two types of absent trials provides a stronger test of the horse-race model. Again, individual subject search rates and intercepts were calculated. There was no difference between the slope values for feature-absent and size-only searches $(t<1.0)$, 
whereas the $y$-intercepts for the two searches differed $[t(11)=3.72, p<.01$, two-tailed $]$. This finding provides strong support for the argument that the search for the features in the both condition proceeds in parallel. ${ }^{5}$

Overall, the pattern of results form the both condition supports the idea that search for two separate features proceeds in parallel, with there being some form of supplementary nonsearch processes invoked after the pickup of the first target. Such processes may be characterized by the switching of attention from the most salient target to the least salient target.

\section{EXPERIMENT 4: TARGET/DISTRACTOR DISCRIMINABILITY}

Experiments 1-3 showed that the difference between performance in the feature- and conjunction-search conditions is not due to the number of features that must be picked up before a response can be made. Experiment 4 examined whether the feature/conjunction search difference arises because of the discrepancy in the number of response-relevant shared features between targets and distractors in the two tasks (for ease of exposition, the phrase shared features will be used henceforth).

There were two conditions, and in each case, subjects responded to a target defined by a conjunction of features. Across the two conditions, the number of shared features was systematically varied. Subjects always had to search displays of colored letters for a same small, green $\mathrm{H}$ (as in Experiments 1-3). However, in contrast to the earlier conjunction searches, the target was defined as a conjunction of three features; namely, shape, size, and color.

In the first (one-feature) condition, the target shared a single feature with each of the distractors. There were three kinds of distractors, and each distractor shared a different feature with the target. In the second (two features) condition, the target shared two features with each of the distractors. Again, three sorts of distractors were used and each shared a different pair of features with the target.

Interestingly, in addition to testing the effects of the number of shared features on conjunction search, Experiment 4 also provided information about how subjects perform when they have to search for a conjunction of three features. Although feature-integration theory is explicit about the two stages of feature extraction and feature integration, the theory is not clear about whether all features are conjoined simultaneously or whether integration proceeds in a piecemeal (feature-by-feature) fashion. If integration operates simultaneously for all the features of a given stimulus, the conjunction search here should be similar to those found in the earlier experiments; however, if integration operates on a feature-by-feature basis, then the slopes of the conjunction search functions should be steeper in Experiment 4.

\section{Method}

Each subject acted in both the one-feature and two-feature conditions in a single experimental session. Half were run on the one- feature condition first, the remaining on the two-feature condition first.

One-feature condition. The displays were made up of the colored letters described previously. The three types of distractors used were medium-sized green As, medium-sized orange $\mathrm{Hs}$, and small orange As.

Two-feature condition. The distractors were small green As, small orange $\mathrm{Hs}$, and medium-sized green $\mathrm{Hs}$.

Apart from the items in the displays, across the two conditions the structure of the stimuli was the same. Four set sizes of distractors were used (i.e., 1, 5, 15, and 30), and the target was added to these distractors on present trials. There were 144 trials in total, with 72 present and 72 absent displays.

Six basic displays were constructed for each display size, and the same six displays were used for present and absent trials. Across the six present displays, the position of the target was varied randomly. The set of six present and absent displays (i.e., six present and absent displays) was repeated three times to give the total set of 18 present and absent responses for each display size.

\section{Results}

Table 8 contains the slope and intercept values for the present and absent search functions in each condition and Figure 7 illustrates the RT search functions.

One-feature condition. Target-present responses departed from linearity $[F(1,11)=8.43, p<.05$, for the quadratic component; $F(1,11)=5.43, p<.05$, for the cubic component]. The error rates were $.9 \%$ for displays of two items, $1.8 \%$ for displays of 6 items, $.9 \%$ for displays of 16 items, and $10.6 \%$ for displays of 31 items. Considered together with the RT data, the error data suggest that subjects tended to trade off accuracy for speed with the largest displays.

Target-absent responses increased linearly with increases in display size $[F(1,11)=58.19, p<.01$, for the linear component]. The speed of search in this condition was approximately $29 \mathrm{msec} /$ item. Furthermore, the slope ratio of the present-to-absent responses was 0.41 , close to the theoretically critical value of 0.5 . Therefore, although the target-present search function departs from linearity, the present-to-absent slope ratio favors a serial, self-terminating search.

Error rates on the absent trials were $1.8 \%$ for displays of one item and $.9 \%$ for the other displays.

Two-feature condition. As in the one-feature condition, RTs on the present trials increased as a function of increasing display size, but the relationship was not strictly

Table 8

Linear Regressions of Reaction Time on Display Size for Experiment 4: Targets Defined by Color, Shape, and Size

Percentage of

Variance

Slope Intercept Accounted For by

\begin{tabular}{lccc} 
Condition & $\begin{array}{c}\text { Slope } \\
(\mathrm{msec} / \mathrm{item})\end{array}$ & $\begin{array}{c}\text { Intercept } \\
(\mathrm{msec})\end{array}$ & $\begin{array}{c}\text { Accounted For by } \\
\text { Linear Component }\end{array}$ \\
\hline $\begin{array}{l}\text { One-Feature Condition } \\
\text { Target-present }\end{array}$ & 11.6 & 531 & $97.2^{*}$ \\
Target-absent & 28.6 & 618 & $99.9^{*}$ \\
Two-Feature Condition & & & \\
Target-present & 37.3 & 612 & $99.4^{*}$ \\
Target-absent & 83.4 & 718 & $99.0^{*}$ \\
\hline
\end{tabular}

*Linear component significant, $p<.01$. 


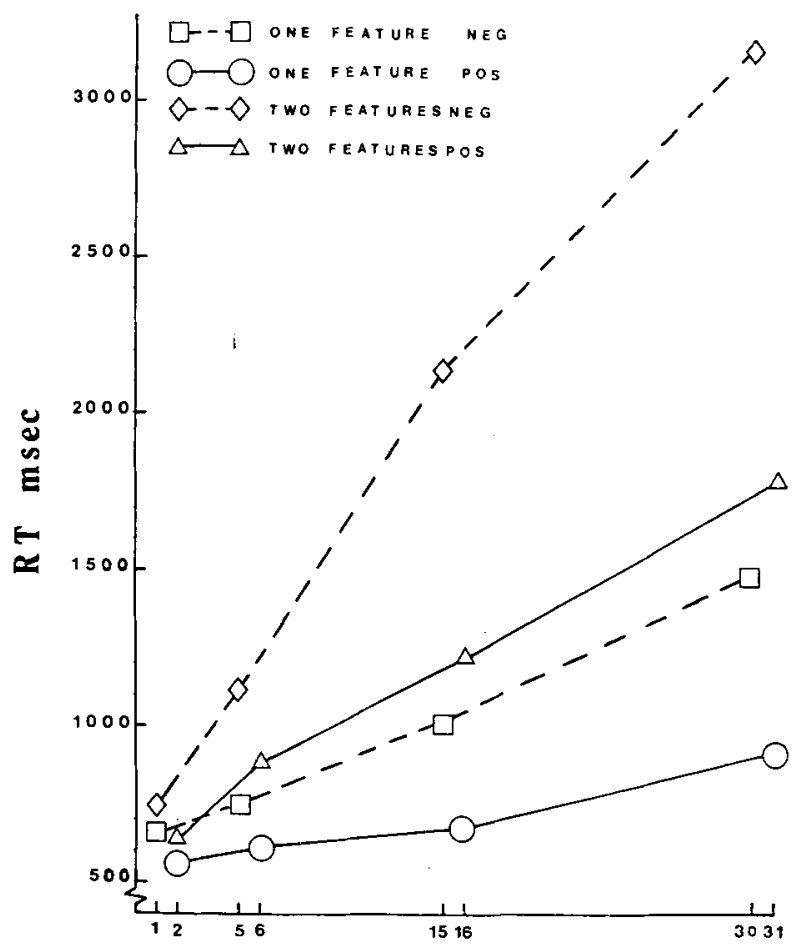

\section{Display Size}

Figure 7. Search times for targets in the one-feature and the twofeature conditions in Experiment 4.

linear $[F(1,11)=13.34, p<.01$, for the cubic component]. The error rates were $.9 \%$ for displays of 2 items, $1.8 \%$ for displays of 6 items, $3.7 \%$ for displays of 16 items, and $14.8 \%$ for displays of 31 items. Again, it appears that subjects tended to trade off accuracy for speed in processing the largest displays.

With absent responses, there was a nonlinear relationship between RTs and increases in display size $[F(1,11)$ $=16.92, p<.01$, for the quadratic component]. From Figure 7 it appears that the RTs to the largest displays were faster than might have been predicted from extrapolating the visible linear function for the smaller displays.

Error rates were $6.0 \%$ for displays of a single item, $12.0 \%$ for displays of 5 items, $4 \%$ for displays of 31 items, and $0 \%$ for displays of 15 items.

Even though both the present and the absent functions for the two-feature conjunction condition are significantly nonlinear, a comparison of the linear components of their slopes reveals a value of .45 , which approximates closely the critical value of .5 . As with the one-feature condition, this is evidence for a serial, self-terminating search.

Comparisons between the one-feature and twofeature conditions. To assess whether the number of shared features affects performance, direct comparisons were carried out across the two conditions. Separate
$2 \times 4$ analyses of variance were carried out on the data from present and absent responses. Condition and display size were entered as fixed factors, and subjects acted as a random factor.

For present responses, the main effects of both condition $[F(1,11)=90.8, p<.01]$ and display size $[F(3,33)$ $=93.65, p<.01]$ were significant, as was the condition $\times$ display-size interaction $[F(3,33)=27.95$, $p<.01]$. The main effect of condition demonstrates that overall responses were slower when the taraget shared two features with the distractors than when it shared only one feature. The effect of display size indicates that RTs increased with increases in display size. Finally, the interaction establishes that the speed of search in the two conditions was significantly different. The speed of search in the two-feature condition was slower than that in the one-feature condition, with the search rate in the twofeature condition being about three times slower.

A similar pattern of results was obtained in the analysis of variance carried out on the target-absent data. The main effects of condition $[F(1,11)=142.94, p<.01]$ and display size $[F(3,33)=155.49, p<.01]$ were significant, as was the condition $\times$ display size interaction $[F(3,33)=75.31, p<.01]$.

\section{Discussion}

The results show that the number of response-relevant shared features between targets and distractors critically determines the speed of search. Search rates for a target that shares two features with each distractor are slower than search rates for a target that shares only one feature, even though both targets may be defined as conjunctions. Search rates for conjunction targets are strongly affected by target-distractor discriminability (see also Treisman et al., 1977).

Interestingly, in both of the conditions, the data were generally consistent with subjects' using a serial, selfterminating search strategy. Target-present searches conform to a linear search pattern, when speed and accuracy are taken into account, and the ratios of the present-absent slopes were close to $1: 2$. Now, in the one-feature condition, targets and distractors differed by two features. This contrasts with the standard conjunction condition in which there are only single feature differences between targets and distractors (e.g., Experiments 1-3, and the twofeature condition in Experiment 4). In this respect, the data indicate that introducing two feature contrasts between targets and distractors is not sufficient to generate parallel search functions. The only instance in which there is a plausible case for departures from a serial search function (i.e., where departures from linearity in the RT data were not traded off with accuracy) is in the absent response data in the two-feature condition. In this condition, the searches at the largest set sizes were faster than would be expected given a strictly linear search. This result is similar to the nonlinear search functions found in the feature-absent and conjunction-absent conditions in 
Experiments 1 and 3, when shape was one of the defining target features. We argued that nonlinear search functions were obtained in Experiments 1 and 3 because subjects were able to search clusters of items rather than individual items. Furthermore, because such clusters are likely to take time to emerge and because absent response times are overall longer than present response times, search based on clustering is likely to be most evident in the absent response data. Such an account may explain the two-feature-absent data here. Indeed, the argument neatly explains why departures from linearity were found only in the two-feature-absent condition, because the search rates in the one-feature condition were considerably faster.

\section{Conjunction Searches in Experiment 4 Relative to Experiments 1-3}

In addition to contrasting performance in the one- and two-feature conditions, Experiment 4 also enabled us to examine visual search performance for conjunctions defined by three features. For instance, a comparison between the search functions in Experiment 4 and Experiments 1-3 should indicate whether the number of features that need to be conjoined for target discrimination influences search efficiency (since in Experiments 1-3 only two features needed to be conjoined).

Table 9 gives the mean correct RTs (in milliseconds) and percentage errors in the conjunction conditions of each experiment, relative to display size.

A series of analyses of variance were carried out on the RT and error data to compare performance in each condition in Experiment 4 with that in the conjunction conditions in Experiments 1-3, for both present and $a b$ sent responses. The error analyses are reported only where

Table 9

Mean RTs (in Milliseconds) and Percentage Errors (PE) for the Conjunction Searches in Experiments 1-3 and the One-Feature and Two-Feature Conditions in Experiment 4

\begin{tabular}{|c|c|c|c|c|c|c|c|c|}
\hline & \multicolumn{8}{|c|}{ Display Size } \\
\hline & \multicolumn{2}{|c|}{2} & \multicolumn{2}{|l|}{6} & \multicolumn{2}{|c|}{16} & \multicolumn{2}{|c|}{31} \\
\hline & Mean & $\mathrm{PE}$ & Mean & $\mathrm{PE}$ & Mean & PE & Mean & PE \\
\hline \multicolumn{9}{|c|}{ "Present" Responses } \\
\hline \multicolumn{9}{|l|}{ Experiment 4} \\
\hline One-feature & 563 & 0.9 & 612 & 1.8 & 678 & 0.9 & 908 & 10.6 \\
\hline Two-feature & 642 & 0.9 & 883 & 1.8 & 1218 & 3.7 & 1759 & 14.8 \\
\hline Experiment 1 & 674 & 4.2 & 958 & 5.1 & 1366 & 10.6 & 1840 & 23.6 \\
\hline Experiment 2 & 699 & 1.4 & 754 & 0.5 & 981 & 8.3 & 1242 & 14.8 \\
\hline Experiment 3 & 657 & 1.3 & 902 & 3.7 & 1327 & 18.9 & 1659 & 25.9 \\
\hline & \multicolumn{8}{|c|}{ Display Size } \\
\hline & \multicolumn{2}{|c|}{1} & \multicolumn{2}{|c|}{5} & \multicolumn{2}{|c|}{15} & \multicolumn{2}{|c|}{30} \\
\hline \multicolumn{9}{|c|}{ "Absent" Responses } \\
\hline \multicolumn{9}{|l|}{ Experiment 4} \\
\hline One-feature & 653 & 1.8 & 759 & 0.9 & 1038 & 0.9 & 1481 & 0.9 \\
\hline Two-feature & 741 & 2.7 & 1113 & 5.5 & 2123 & 0.0 & 3148 & 1.8 \\
\hline Experiment 1 & 709 & 3.7 & 959 & 3.2 & 1856 & 7.8 & 2628 & 6.4 \\
\hline Experiment 2 & 830 & 2.8 & 882 & 0.9 & 1313 & 1.4 & 1805 & 2.3 \\
\hline Experiment 3 & 698 & 5.1 & 1159 & 3.2 & 2218 & 3.7 & 2950 & 0.9 \\
\hline
\end{tabular}

they add substantially to the RT data. The results may be summarized as follows:

1. The one-feature condition of Experiment 4 tended to be easier than each of the conjunction conditions in Experiments 1-3. This effect was manifest in terms of the speed of search.

For target-present responses, there were interactions between the main effects of condition and display size in each analysis of the RTs $[F(3,33)=23.82,6.94$, and 24.33 , all $p s<.001$, for comparisons between the onefeature condition and the conjunction conditions in Experiments 1,2 , and 3 , respectively].

For target-absent responses, the condition $\times$ display size interaction was significant in the comparisons between RTs in the one-feature condition of Experiment 4 and the conjunction conditions in Experiments 1 and $3[F(3,33)$ $=23.82$ and $24.47, p s<.001$, respectively] .

2. Search rates in the two-feature condition of Experiment 4 tended to be slower than those in the conjunction condition in Experiment 2.

There were reliable condition $x$ display size interactions both when the target was present $[F(3,33)=12.12$, $p<.01]$, and when it was absent $[F(3,33)=30.55$, $p<.001]$.

3. The conjunction conditions in Experiments 1 and 3 tended to be more difficult than the two-feature condition in Experiment 4.

For "present" responses, there were no differences in the RT data, but there were in the error data. More errors were made in the conjunction condition of Experiment 1 than in the two-feature condition $[F(1,11)=7.7$, $p<.025]$, but there was no condition $\times$ display size interaction $(F<1.0)$. More errors were also made in the conjunction condition in Experiment 3 than in the twofeature condition, and this effect increased as a function of display size $[F(1,11)=9.49$ and $F(3,33)=5.39$, both $p$ s $<.01]$.

For "absent" responses, a more complex picture emerges. Relative to the two-feature condition, search rate was faster in Experiment $1[F(1,11)=3.10, p<.05]$, but more errors were made $[F(3,33)=5.46, p<.01$, for the condition $\times$ display size interaction]. Thus there is no clear difference between the two conditions. More errors were made in Experiment 3 than in the two-feature condition $[F(3,33)=3.48, p<.05$, for the condition $\times$ display size interaction], and in this case, there was no effect of the condition on RTs.

The main conclusion from the above comparisons is that performance is not necessarily less efficient when three rather than two features need to be conjoined for target discrimination. Three features needed to be conjoined in both of the conditions in Experiment 4 . However, performance was more efficient in the one-feature condition of that experiment than in any of the conjunction conditions in Experiments 1-3, in which only two features needed to be conjoined (point 1 above). Also, performance tended to be more efficient in the two-feature condition 
of Experiment 4 than in the conjunction condition of Experiments 1 and 3 (point 3 above). It seems clear that subjects do not integrate feature information in a piecemeal (feature-by-feature) fashion to discriminate targets from distractors. Integration operates simultaneously for all the features in a given object.

\section{GENERAL DISCUSSION}

Our initial aim was to contrast visual search for one and two targets defined by a single feature difference relative to their background (with the single- and both-feature conditions), and to investigate the effects of the number of relevant shared features between targets and distractors on search for a conjunction target. The data were informative on both of these issues. We will deal with the main results from the both and the conjunction search conditions in turn, before proceeding to give an account of the data.

\section{Both Searches}

Search for two feature targets (in the both condition in Experiments 1-3) differs from search for simple feature targets only in the intercept of the search functions; search rates do not differ. The search pattern for the two feature targets does not correspond to the serial search pattern observed for a single target defined by a conjunction of features (Experiments 1-3). This is supported by the slope values of the corresponding functions. Although the slopes of the search functions for absent versus present responses always approximated 2:1 in the conjunction conditions, the corresponding slope ratios were considerably larger in all the feature conditions (i.e., for single-feature and both-feature targets), even when the feature-present searches were themselves linearly related to the display size (Experiments 1 and 3).

The data from the both condition (Experiments 1-3) are consistent with the proposal that subjects are able to conduct simultaneous, spatially parallel searches for targets defined by two different types of feature, and that this can occur even when those searches are relatively inefficient (e.g., as in Experiment 3). However, the detection of targets made available by these searches does not occur in parallel; some serial process is involved in making correct detections to two-feature targets, as evidenced by the intercept increases in the search functions relative to the single-feature conditions. It is interesting to note that the difference between the both and the single-feature conditions occurred here even though separate responses to the two features were not required in the both condition. This implicates a central decision mechanism as the locus of the difference, and extends previous studies that have examined the detection of multiple targets where subjects were required to make different decisions regarding the available targets (see Duncan, 1980).

This result is inconsistent with the notion that a focal attentional process can in some way be bypassed when single-feature targets are detected (cf. Treisman \& Gelade,
1980). Detection responses, even to feature targets, appear to demand focal attention (see also Duncan, 1984, 1985). To account for the intercept effects found in the both condition in each experiment, we may suggest that, following the detection of one type of feature target, attention must be switched to the more slowly discriminated target. Such switching appears to take time, but it is not affected by the number of irrelevant distractors. ${ }^{6}$

It is possible to sketch at least two different accounts of switching. For instance, when subjects search for both a blue target and another target defined by its shape (say a $\mathrm{C}$ relative to As and $\mathrm{Hs}$; cf. Experiment 1 here), the color-defined target will typically be detected more rapidly than the shape-defined target. We suggest that the detection of either target requires attention. Thus, in this instance, subjects will first attend to the color-defined target and then to the shape-defined target. However, different kinds of representations can be assumed to underlie this attentional act. One possibility is that the representation of an attended target makes explicit all of its visual attributes (e.g., its size, shape, and location in addition to its color; see Duncan, 1984). An alternative is that the target is coded in terms of its value only within the dimension that supports the discrimination (i.e., initially, color, and then shape). These two accounts, which may be termed the object-switching and the dimensionswitching accounts, make different predictions. According to the dimension-switching account, it may be possible for attention to be switched more easily between some dimensions than between others. The test of this account would be to examine differences in intercept values between various search functions when the feature target takes on different critical dimensions. The objectswitching account does not make this prediction, since switching will always operate on a complete specification of the object's attributes. This account makes no claims about difficulties in processing particular dimensions. Unfortunately, the present data are merely suggestive on this issue. The intercept increases in the both condition relative to the slowest feature condition tended to be greatest when color was the "fast" feature. For example, in Experiment 1, when shape and color defined the targets, the average intercept increase (over subjects) was $149 \mathrm{msec}$ in the both-present condition relative to the shape-present condition; when shape and size defined targets (Experiment 3 ), the average intercept increase was $98 \mathrm{msec}$ (again, relative to the shape-present condition). These differences were not significant $(t<1.0)$. Nevertheless, it would be interesting to examine the theoretical possibility in more detail, using a wider range of features and a more sensitive within-subjects design.

We may also question the constraints governing the ability to search simultaneously for two different featuredefined targets. The present data indicate that subjects can search simultaneously for two features defined along dimensions of shape, size, and color. It is by no means clear, though, whether we can also search for two features specified within each dimension (say, a $\mathrm{C}$ and an 
$\mathrm{X}$, or a blue and a white target). This issue is, of course, relevant to the question of whether attention is switched between objects or between dimensions in feature-search tasks. It is also relevant to the question of whether we may process in parallel, and without mutual interference, two or more stimuli with different values within a given dimension (see Allport, 1971; Duncan, 1985).

\section{Conjunction Searches}

Experiment 4 demonstrated that conjunction searches remain serial even when targets and distractors differ by two features (in the one-feature condition). This suggests that discriminability per se is not the factor critically determining the differences between the search for featuredefined and conjunction-defined targets: targets were more discriminable from distractors in the one- relative to the two-feature condition in Experiment 4, yet in both cases serial searches were found. It appears, then, that targetdistractor discriminability influences the rate of conjunction search without changing its nature (see also Treisman et al., 1977). Search was also not constrained by the number of features that needed to be conjoined for discrimination to occur, since performance was no more difficult when targets were defined in terms of three features (in Experiment 4) than when they were defined in terms of two features (see, in particular, Experiments 1 and 3).

This leaves us with the question of which factors determine conjunction searches. Perhaps the most illuminating comparison in this respect is that between the conjunction conditions and the both conditions. The primary procedural difference between these conditions was that in the conjunction conditions, performance depended on the detection of two features in the same spatial location. It seems that this constraint is critical in determining that visual search is serial and self-terminating.

Now, we may hypothesize that the separate dimensions of the present stimuli are represented in independent, retinotopic maps (see Cowey, 1979; Treisman \& Souther, 1985). To detect a conjunction target, any activation within each of these maps would need to be coordinated on the basis of location information. In contrast, responses in the both condition do not require coincident activity in the same locations in two or more different maps; activity within a given map would be a logically sufficient criterion for a response. It follows that conjunction searches are constrained to operate serially because detection depends on coincident activity in different feature maps. Why should this be so?

One possibility is that activity within different feature maps may be correctly integrated only by the serial application of an attentional "spotlight" to activated locations in the feature maps (e.g., Treisman \& Gelade, 1980; Treisman \& Souther, 1985). On the other hand, the detection of single features will depend solely on their being represented in an associated feature map; it will not be affected by the operation of focal attention (Treisman \& Souther, 1985). This argument, then, maintains that the contrast between visual search for feature-defined and conjunction-defined targets reflects qualitative differences in the role of focal attention.

Against the above argument, the present finding of intercept effects on search for two relative to one feature target (in the both condition) indicates the involvement of focal attention even in the detection of feature targets.

An alternative account is that visual search is constrained primarily by the fidelity of the information required to support the discrimination. The notion of fidelity here extends beyond that of discriminability within a single dimension (such as differences in hue, size, or shape) to include accuracy of location coding. Conjunction searches are constrained by the fidelity of location coding, whereas feature searches are constrained by the fidelity of the coding of attributes within the relevant dimension(s). For instance, a visual display composed of colored letters may be described at various levels. When the whole display is taken as being a single perceptual object, "part" information will correspond to regions containing subgroups of letters. Coding at this level will be sufficient to support the discrimination of targets defined by salient featural differences relative to the background. However, information concerning the visual characteristics of individual letters (their exact location, size, shape, and even color) may not be accurately coded, since such information is coded across regions of the display. At another level of description, letters may be coded as individual perceptual objects. Now "part" information corresponds to the visual characteristics of individual letters. It follows that discriminations based on the accurate coding of such characteristics must operate on descriptions of single letters. This will give rise to strictly serial, selfterminating search functions.

The fidelity account can accommodate differences between conjunction and feature searches. Searches for a conjunction-defined target will be serial and selfterminating because accurate location coding will always require representation at the level of individual letters. Note that this type of search should occur irrespective of the discriminability of other visual characteristics of the stimuli (although discriminability within other dimensions will affect the rate of search; cf. Experiment 4 here).

The fidelity account of feature search is rather more complex. In many cases, searches for feature-defined targets will show evidence of spatially parallel processing because the target feature is specified at levels of description higher than the individual stimuli (up to and including the whole display). However, the account also predicts that a family of search functions may be generated, from flat nonlinear searches to strictly serial, self-terminating searches, according to the discriminability of the target feature within the relevant dimension. As the difficulty of the feature discriminations increases, search functions should increasingly approximate those corresponding to serial, self-terminating search.

Some evidence consistent with the latter argument comes from a control study we conducted to check on the 
shape discrimination required in all the present conjunction search tasks, namely, A versus $H$. The design of the stimuli followed that used in the earlier experiments; however, the distractors were always small green As and the target was the small green $\mathrm{H}$. Six subjects were run in this experiment, which was procedurally the same as all others reported here. Averaging across the subjects, the mean RTs on target-present trials were 736, 1,104, 1,733 , and $2,537 \mathrm{msec}$ at each respective display size; the corresponding error rates were $2.7 \%, 8.3 \%, 12.0 \%$, and $29.6 \%$, respectively. On target-absent trials, the mean RTs were 733, 1,426, 2,710, and 3,698 $\mathrm{msec}$ and the corresponding error rates were $4.6 \%, 2.7 \%, 2.7 \%$, and $2.7 \%$, respectively. There was a reliable linear relationship between target-present responses and display size $[F(1,5)=65.77$ for the RT data, and $F(1,5)=25.91$ for the error data; both $p s<.01]$. Absent responses departed from linearity. For the RT data, there was a reliable quadratic trend $[F(1,5)=10.94, p<.05$; for the error data, no component reached significance]. The search rate for target-present responses was $60.8 \mathrm{msec} / \mathrm{item}$; for target-absent responses, it was $100.3 \mathrm{msec} / \mathrm{item}-\mathrm{a}$ search ratio of .61 . Search for the shape-defined target here was clearly difficult, and the search rate was the slowest of any of the present experiments. More importantly, the search functions correspond more closely to those expected from a serial and selfterminating search than any of the feature-search functions in Experiments 1-3, at least if we take the presentabsent slope ratios as indicative of the search process.

Other evidence that difficult feature discriminations may be based on a serial, self-terminating search comes from data reported by Treisman and Souther (1985). In their Experiment 4, subjects had to detect the presence of a complete circle (the target) against a background of incomplete circles (i.e., circles with gaps acted as the distractors). When the distractors contained small gaps, the search functions were linear and the present-absent slope ratio was close to .5 (it was .492 in the "medium" gap condition, but .783 with a "small" gap). When the distractors contained large gaps (when they were semicircles), there was only a small effect of display size on performance and the present-absent slope ratio no longer approximated to .5 (it was .2). These data suggest that searches for single features become serial as discriminability within the relevant dimension decreases.

The fidelity account thus differs from feature integration theory in that it holds that search for single-featureand conjunction-defined targets do not differ qualitatively, since equivalent (serial) search functions will be generated if the feature discrimination is made sufficiently difficult. We suggest that the critical factor underlying different visual search functions is the level at which a display is described. Descriptions at the level of the whole display produce parallel search functions; descriptions at the level of individual display items produce serial search functions. Attention may be described as the process of selecting between the different levels of visual descrip- tion of a display, which may be made available in parallel but over different durations. Performance will usually be determined by the highest level of description that supports discrimination.

\section{REFERENCES}

Allport, D. A. (1971). Parallel encoding within and between elementary stimulus dimensions. Perception \& Psychophysics, 10, 104-108. Allport, D. A., TIPPER, S. P., \& ChMiel, N. R. J. (1985). Perceptual integration and postcategorical filtering. In M. I. Posner \& O. S. M. Marin (Eds.), Attention and performance XI. Hillsdale, NJ: Erlbaum.

ATtNeAve, F. (1950). Dimensions of similarity. American Journal of Psychology, 63, 516-556.

Bundesen, C., \& Pedersen, L. F. (1983). Color segregation and visual search. Perception \& Psychophysics, 33, 487-493.

CowEY, A. (1979). Cortical maps and visual perception. Quarterly Journal of Experimental Psychology, 31, 1-17.

DUNCAN, J. (1980). The locus of interference in the perception of simultaneous stimuli. Psychological Review, 87, 272-300.

DUNCAN, J. (1984). Selective attention and the organization of visual information. Journal of Experimental Psychology: General, 113, 501-517.

DunCan, J. (1985). Visual search and visual attention. In M. I. Posner \& O. S. M. Marin (Eds.), Attention and performance XI. Hillsdale, NJ: Erlbaum.

Egeth, H. E., Virzi, R. A., \& Garbart, H. (1984). Searching for conjunctively defined targets. Joumal of Experimental Psychology: Human Perception \& Performance, 10, 32-39.

GARNER, W. R. (1974). The processing of information and structure. Potomac, MD: Erlbaum.

HANDEL, S., \& LMA, S. (1972). The free classification of analyzable and unanalyzable stimuli. Perception \& Psychophysics, 12, 108-116.

Humphreys, G. W., RidDoch, M. J., \& Quinlan, P. T. (1985). Interactive processes in perceptual organization: Evidence from visual agnosia. In M. I. Posner \& O. S. M. Marin (Eds.), Attention and performance XI. Hillsdale, NJ: Erlbaum.

MiNSKY, M. (1975). A framework for representing knowledge. In P. H. Winston (Ed.), The psychology of computer vision. New York: McGraw-Hill.

Ostry, D., Moray, N., \& Marks, G. (1976). Attention, practice, and semantic targets. Journal of Experimental Psychology: Human Perception \& Performance, 2, 326-336.

STERNBERG, S. (1975). Memory scanning: New findings and current controversies. Quarterly Journal of Experimental Psychology, 27, 1-32.

Treisman, A. M. (1982). Perceptual grouping and attention in visual search for features and for objects. Journal of Experimental Psychology: Human Perception \& Performance, 8, 194-214.

Treisman, A. M., Gelade, G. (1980). A feature-integration theory of attention. Cognitive Psychology, 12, 97-136.

Treisman, A. M.,. \& SCHMIDT, H. (1982). Illusory conjunctions in the perception of objects. Cognitive Psychology, 14, 107-141.

Treisman, A. M., \& Souther, J. (1985). Search asymmetry: A diagnostic for preattentive processing of separable features. Journal of Experimental Psychology: General, 114, 285-310.

Treisman, A. M., SyKes, M., \& Gelade, G. (1977). Selective attention and stimulus integration. In S. Dornic (Ed.), Attention and performance VI. Hillsdale, NJ: Erlbaum.

\section{NOTES}

1. Since at least one distractor was always present, it is possible that subjects could have responded "present" on detecting two stimuli in the displays and "absent" on detecting only one. However, it is unlikely that subjects would use a similar strategy with the larger displays.

2 . In determining the significance of orthogonal components, the most sensitive method was used. Although it is possible to test the compo- 
nents using the overall condition error term in the corresponding $F$ ratios, separate error terms for each component were derived and used. This is in keeping with the repeated measures design.

3. In all cases in which the present/absent search ratios are used to determine whether search was parallel or serial and self-terminating, further analyses of variance (ANOVAs) were conducted to test the ratios against the value of 0.5 . In these ANOVAs, RTs for present responses were doubled and compared with absent RTs. For the feature and both searches, which gave linear target-present functions (i.e., Experiments 1 and 3), there were interactions between display size and target presence/absence $[F(3,33)=17.02, p<.001$, and $F(3,33)=$ $4.93, p<.01$, respectively]. This confirms that the present/absent slope ratios were less than 0.5 . For all the conjunction searches, bar that in Experiment 1, display size effects combined additively with target presence/absence [for Experiment 2, $F<1.0$; for Experiment 3, $F(3,33)=2.23, p>.05$; for the one-feature condition $F(3,33)=2.62$, $p>.05$; and for the two-feature condition, $F(3,33)=1.58, p>.05]$. In Experiment 1, there was a display size $\times$ target present/absent interaction for the conjunction search $[F(3,33)=4.91, p<.001]$. This interaction went in the direction opposite to that predicted by parallel search: "absent" responses were faster than might have been expected if search were solely serial and self-terminating. This result is consistent with the claim that, on absent trials, subjects search local clusters rather than individual items (see Discussion of Experiments 1,2, and 3).

4. It is also interesting to note the contrast between the search rate for shape in Experiment 3 and that for shape in Experiment 1. The same shape (i.e., C) was used as a target in the two experiments, but the search rate in Experiment 3 is approximately twice that for shape in Experiment 1 . An explanation for this difference stems from the size manipulation used in Experiment 3. In Experiment 1, the shape targets were small orange or green Cs. In Experiment 3, the shape targets were green medium or small Cs. When the data for the trials with small and medium $\mathrm{Cs}$ in Experiment 3 were separated out, the search times showed more of a systematic increase as a function of display size for small rather than medium Cs. For small Cs, mean search times for the four display sizes were $571,577,656$, and 711 msec, respectively. For medium Cs, the corresponding means were 556, 553, 550, and $606 \mathrm{msec}$. In averaging over the responses to the two types of target, the search rate for shape is faster than might have been predicted from the results of Experiment 1 simply because medium Cs were more discriminable from their distractors than small orange Cs.

5. The tests of the intercepts and the slopes of the search functions assume that the data are adequately "captured" by linearity. Note here that most of the departures from linearity are, in fact, quite small. Also, we have conducted matching ANOVAs, where the both and the slowest feature searches have been directly compared across the display sizes. In no case have we found an interaction between the display size and the difference between the both and the slowest feature searches, although the overall speed of search in the both condition is slower. This is consistent with an effect on the intercept, but not on the slope. Such analyses stand even with nonlinear search functions, providing there are main effects of display size in each of the conditions.

6. The assumption of feanure-integration theory, that single features can be detected in parallel, can be maintained if it is also assumed that the intercept effect in the both condition reflects the additional time to check detected features against a memory set of possible targets. Now, the memory set size of possible targets in the both and single-feature conditions were the same (i.e., two). Thus, the difference between these two conditions would be reflected in the memory comparison time. However, any differences due to the contrasting memory comparison times required for two items (in the both condition) relative to one item (in the single-feature condition) are likely to be too small to account for the data. Memory comparison times here can be taken to be of the order of, at the most, $40 \mathrm{msec} /$ item (see Sternberg, 1975). If we consider only Experiment 1, the differences in RTs to the "fast" and the "slow" features (i.e., color and size) were of the order of $250 \mathrm{msec}$ at the display size of 30 . In the both condition, this would allow ample time for subjects to compare and remove the "fastest" feature from the memory set prior to detecting the "slower" feature. This would reduce performance in the both condition to that in the single-feature condition when shape was the target. This makes the incorrect prediction that performance in the both and the shape-present condition should not differ.

(Manuscript received May 21, 1986; revision accepted for publication February 2, 1987.) 